\title{
Identification of Constituents and Exploring the Mechanism for Toutongning Capsule in the Treatment of Migraine
}

\author{
Xia Du $\mathbb{D}^{1,2}$ Zhibiao Di, ${ }^{1}$ Yang Liu, ${ }^{1}$ Wenbing Zhi, ${ }^{1}$ Yuan Liu, ${ }^{1}$ Hong Zhang $\mathbb{D}^{1}{ }^{1}$ \\ and Feng Liu $\mathbb{D}^{3,4}$ \\ ${ }^{1}$ Institute of Traditional Chinese Medicine, Shaanxi Academy of Traditional Chinese Medicine, Xi'an, Shaanxi 710003, China \\ ${ }^{2}$ Center for Post-Doctoral Studies, China Academy of Chinese Medical Sciences, Beijing 100700, China \\ ${ }^{3}$ Shaanxi Institute of International Trade \&Commence, Xi'an 712046, China \\ ${ }^{4}$ Shaanxi Buchang Pharmaceutical Co. Ltd, Xi'an 710075, China
}

Correspondence should be addressed to Hong Zhang; zhanghong919919@163.com and Feng Liu; liufeng1720@163.com

Received 14 January 2021; Revised 4 November 2021; Accepted 23 November 2021; Published 15 January 2022

Academic Editor: Mohammad Fattahi

Copyright (c) 2022 Xia Du et al. This is an open access article distributed under the Creative Commons Attribution License, which permits unrestricted use, distribution, and reproduction in any medium, provided the original work is properly cited.

\begin{abstract}
Toutongning capsule (TTNC) is an effective and safe traditional Chinese medicine used in the treatment of migraine. In this present study, a multiscale strategy was used to systematically investigate the mechanism of TTNC in treating migraine, which contained UPLC-UESI-Q Exactive Focus network pharmacology and experimental verification. First, 88 compounds were identified by the UPLC-UESI-Q Exactive Focus method for TTNC. Then, the target fishing for these compounds was performed by means of an efficient drug similarity search tool. Third, a series of network pharmacology experiments were performed to predict the key compounds, targets, and pathways. They were protein-protein interaction (PPI), KEGG pathway enrichment analysis, and herbs-compounds-targets-pathways (H-C-T-P) network construction. As a result, 18 potential key compounds, 20 potential key targets, and 6 potential signaling pathways were obtained for TTNC in treatment with migraine. Finally, molecular docking and experimental were carried out to verify the key targets. In short, the results showed that TTNC is able to treat migraine through multiple components, multiple targets, and multiple pathways. This work may provide a theoretical basis for further research on the molecular mechanism of TTNC in the treatment of migraine.
\end{abstract}

\section{Introduction}

Migraine is a common chronic and paroxysmal neurological disease. The most characteristic symptoms associated with migraine include nausea, emesis, photophobia, and phonophobia. In addition, patients tend to have a variety of other neurological symptoms, such as dizziness, tinnitus, and cognitive impairment $[1,2]$. Epidemiological survey results showed that the overall incidence of migraine in the general population was $12 \%$, and the annual cost of migraine was estimated to be more than US $\$ 20$ billion. Plus, the WHO ranked migraine as the third most prevalent medical condition and the second most disabling neurological disorder in the world [3-5]. In recent years, many diagnostic guidelines for migraine have shown up and played an important role in its prevention and treatment. However, due to the high incidence of migraine, high disability, high missed diagnosis rate, and low control rate, the current diagnosis and treatment outcome of migraine are still not ideal. Acute treatment of migraine includes the use of drug therapy, from ergots and triptans to nonsteroidal anti-inflammatory drugs $[6,7]$. It is known that triptans have been the leading evidence-based, acute therapies for migraine for the past 20 years, including sumatriptan, naratriptan, rizatriptan, zolmitriptan, eletriptan, almotriptan, frovatriptan, and sumatriptan/ naproxen combination $[8,9]$. These FDA-approved drugs affect serotonin and have proven efficacy in the acute treatment of migraine. Nevertheless, most of all these drugs were short-acting. Also, they were contraindicated in coronary artery disease, uncontrolled hypertension, 
and Prinzmetal angina. In addition, preventive treatment medications can reduce the frequency, severity, and duration of attacks in people with frequent migraine, such as beta-blockers (propranolol and metoprolol), calcium channel blockers (flunarizine), antiepileptics (sodium valproate, topiramate, lamotrigine, and gabapentin), and antidepressants (amitriptyline, fluoxetine, and sertraline) [10-12]. Depressingly, the long-term use of these drugs may result in adverse effects such as lethargy, fatigue, anxiety, depression, and muscle soreness.

Traditional Chinese medicine (TCM) has been widely used in the treatment of migraine for more than 2000 years. Migraine belongs to the category of Shou Feng or Nao Feng in TCM, which has been recorded as early as in the Yellow Emperor's Medicine Classic (Chinese name in pinyin Huang Di Nei Jing), a classical literature from the Han Dynasty (AD 25-220). In TCM, "wind, heat, phlegm, and blood stasis" are considered to be the main pathogenic factor of migraine. In this regard, the critical points were extinguishing wind and resolving phlegm activate blood and relieving stasis in the treatment of migraine. Toutongning capsule (TTNC) functions calming wind, clearing phlegm, removing blood stasis, and relieving pain. Hence, Toutongning capsule is frequently used in the treatment of migraine in clinical and has a significant effect. The components of the formula include Smilacis Glabrae Rhizoma, Gastrodiae Rhizoma, Polygoni Multiflori Radix, Angelicae Sinensis Radix, Saposhnikoviae Radix, and Scorpio. Among these herbs, Gastrodiae Rhizoma is able to calm the liver to stop endogenous wind and combined with Smilacis Glabrae Rhizoma can pacify the liver to subdue yang. Polygoni Multiflori Radix has the effect of replenishing lean and nourishing blood. Angelicae Sinensis Radix can nourish blood and activate blood. Saposhnikoviae Radix is able to dispel wind and eliminate dampness. Scorpio can break blood, expel stasis, and free the collateral vessels to relieve pain. The results of evidence-based medicine research showed that the effective rate of TTNC was $72.5 \%$, with a prevention rate of $86.5 \%$. No adverse events took place for the treatment of 400 patients with primary migraine by a randomized, double-blind, placebo-controlled multicenter clinical trial [13]. At present, most research studies on TTNC tended to focus on clinical research. There are few studies on the molecular mechanism based on the multiple components and multiple targets of TTNC.

In this present study, an integrated strategy was used to investigate the mechanism of TTNC in treating migraine, which contained UPLC-UESI-Q Exactive Focus and network pharmacology. The UPLC-UESI-Q Exactive Focus method was adopted to identify the chemical composition for TTNC. The technologies of network pharmacology $[14,15]$ were performed for clarifying the potential mechanism of action for TTNC in treatment with migraine, such as the target fishing, protein-protein interaction (PPI), KEGG pathway enrichment analysis, and herbs-compounds-targets-pathways (H-C-T-P) multidimensional network construction (Figure 1). This present study may provide a theoretical basis for further research on the molecular mechanism of TTNC in the treatment of migraine.

\section{Materials and Methods}

\subsection{The Chemical Composition Analysis by UPLC-UESI-Q Exactive Focus}

2.1.1. Chemicals and Materials. Acetonitrile (HPLC-grade) and methyl alcohol (HPLC-grade) were obtained from Fisher Scientific Company. The MS-grade formic acid was obtained from Sigma Aldrich (Germany) Trading Co., Ltd. Toutongning capsule (TTNC) (batch no. 190312) was provided by Buchang Pharmaceuticals (Xi'an, China).

2.1.2. UPLC-UESI-Q Exactive Focus Conditions. The instrument used for the chromatographic separation was a Thermo Scientific Q Exactive Focus system coupled with the Thermo Scientific Ultimate 3000 system [16, 17]. Separation was performed on an Accucore aQ C18 column $(150 \mathrm{~mm} \times 2.1 \mathrm{~mm}, 2.6 \mu \mathrm{m})$. In addition, column temperature was maintained at $30^{\circ} \mathrm{C}$. The mobile phase consisted of two solvents A ( $0.1 \%$ formic acid in water) and B (methyl alcohol) at a flow rate of $0.30 \mathrm{~mL} / \mathrm{min}$. A linear gradient elution of $\mathrm{A}$ and $\mathrm{B}$ was used according to the following program (0-10 min, 5-25\% B; 10-25 min, 25-60\% B; 25-30 min, 60\% B; 30-35 min, 60-95\% B; 35-37 min, 95-5\% $\mathrm{B}$; and $37-40 \mathrm{~min}, 5 \% \mathrm{~B})$. The injection volume was set $3 \mu \mathrm{L}$. Liquid chromatography HESI-MS analysis was conducted by means of the positive and negative electrospray model. The mass spectral scan ranges from $80 \mathrm{Da}$ to $1200 \mathrm{Da}$. The spray voltage was set at $3.5 \mathrm{kV}$. Besides, the other conditions are shown as follows: capillary temperature, $320^{\circ} \mathrm{C}$; Aux gas heater temperature, $350^{\circ} \mathrm{C}$; sheath gas flow rate, $40 \mathrm{arb}$; Aux gas flow rate, $10 \mathrm{arb}$; S-lens RF level, $50.0 \mathrm{~V}$; resolution, full MS 70000, dd-MS2 35000; and scanning mode, full MS-ddMS2.

\subsection{The Mechanism of Toutongning Capsule (TTNC) in the Treatment of Migraine by Means of Network Pharmacology}

2.2.1. Target Fishing of the Chemical Composition for TTNC. The potential targets of the chemical composition by UPLCUESI-Q Exactive Focus for TTNC were predicted in an encyclopedia of traditional Chinese medicine (ETCM) platform using MedChem Studio (version 3.0), which is an efficient drug similarity search tool to identify the similarity between the known drugs in the DrugBank database with the tested compounds $[18,19]$. The similarity is manifested by the Tanimoto score, and the Tanimoto score is in the range of $(0,1)$, where " 0 " denotes completely different structures between ingredients and known drugs, and " 1 " denotes 


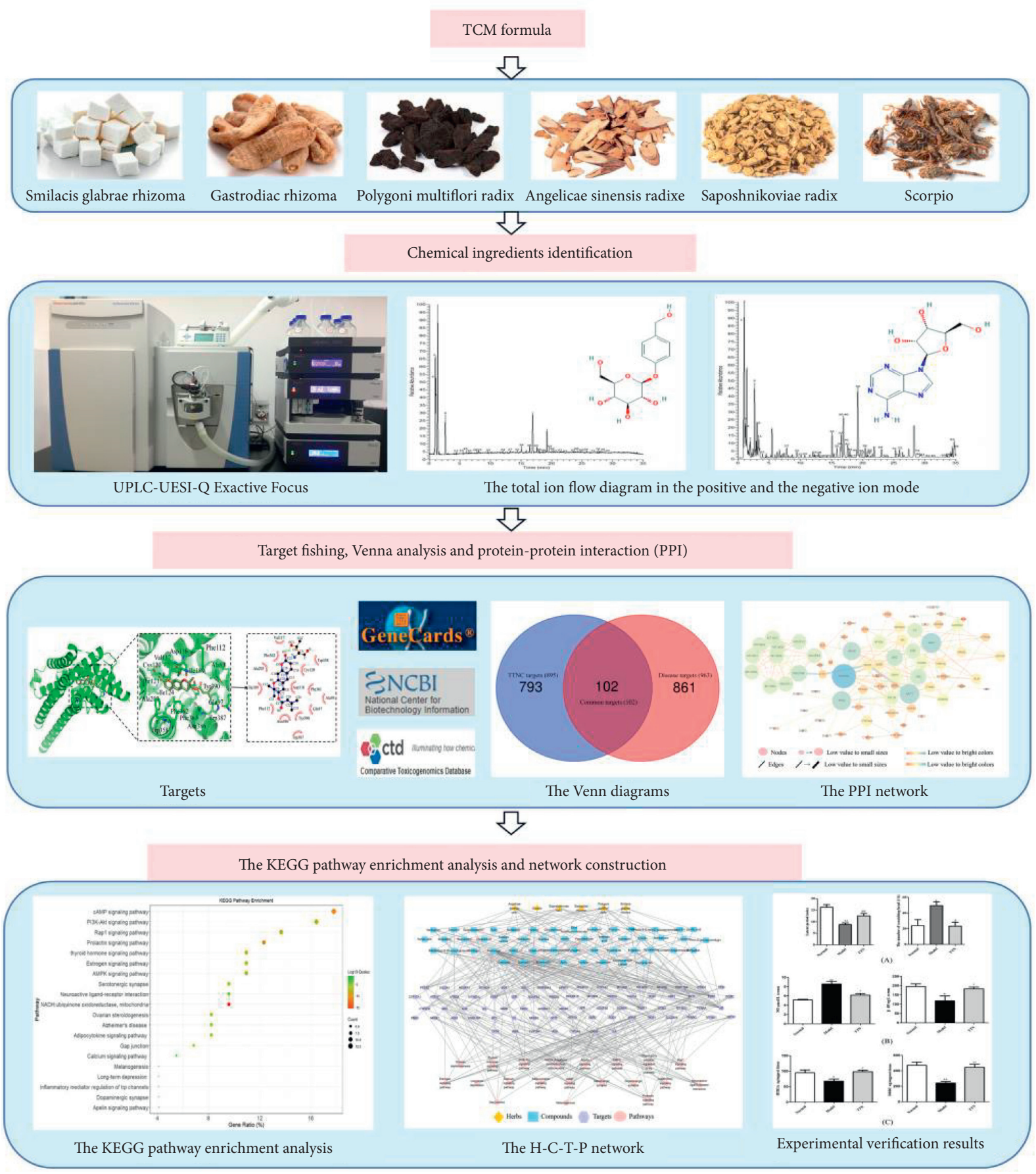

FIgURE 1: The detailed flowchart for TTNC in the treatment of migraine.

identical structures of two components. In this present study, the threshold of the Tanimoto scores was set to 0.75 .

\subsubsection{The Targets Related to the Migraine. The targets related} to the migraine were collected from the GeneCards database. The targets with relevance score above the average were regarded as the migraine targets. Relevance score is the Novoseek score of the relevance of the disease to this gene based on their literature text-mining algorithms [20]. All targets were converted by means of UniProt database and corrected to the official shorthand [21].
2.2.3. The Targets of the Chemical Composition for TTNC in the Treatment of Migraine. The nonhuman targets were deleted from all the component targets and disease-causing genes. After that, Venn online tool free software (https:// bioinformatics.psb.ugent.be/webtools/Venn/) was used to screen the overlap of disease-causing genes and component targets for TTNC.

2.2.4. Protein-Protein Interaction (PPI) Network Construction and Core Target Screening. The targets were introduced into the online system public database STRING (Search Tool 
for Known and Predicted Protein-Protein Interactions, version 11.0, https://string-db.org/) to obtain the interaction between proteins and predicted proteins [22]. The protein type was set to "Homo sapiens," and the minimum interaction threshold with the highest confidence was -0.900 , and the targets with poor connection of the primary network were removed. After then, the PPI network was constructed using Cytoscape 3.7.2, and the network topological properties were analyzed for each node using the CytoNCA plugin for Cytoscape 3.7.2, such as degree centrality (DC), betweenness centrality (BC), and closeness centrality (CC) [23].

2.2.5. KEGG Pathway Analysis. The biological functions and participated pathways of the candidate drug targets have been investigated according to KEGG (Kyoto Encyclopedia of Genes and Genomes) pathway analysis by means of DAVID (Database for Annotation Visualization and Integrated Discovery) $[24,25]$.

\subsubsection{The Herbs-Compounds-Targets (H-C-T-P) Network} Construction and Analysis. The H-C-T-P network was constructed for TTNC in the treatment of migraine by Cytoscape 3.7.2. The network topological properties, including DC, BC, and CC, were analyzed by the NetworkAnalyzer plugin in the software to explore the key compounds and targets for TTNC in the treatment of migraine.

2.3. Molecular Docking. Molecular docking was performed to investigate the interactions between some key components and targets. The structures of the key components were obtained from the PubChem database (https://pubchem. ncbi.nlm.nih.gov). The structures of the targets were obtained from the PDB database (https://www.pdb.org). The docking simulation was performed by means of AutoDock 4.2.6 software. During the docking calculations, hydrogen atoms and Gasteiger charges were added to the protein using the automated docking tool. The auxiliary program AutoGrid was used to set the docking boxes. These docking boxes were defined according to the crystal structures of complex of the proteins with known ligands. The Lamarckian genetic algorithm (LGA) was adopted for each docking progress.

\subsection{Experimental Verification}

2.4.1. Animals. Sprague-Dawley (SD) rats $(n=24), 6$ weeks, weighing 180-220 g, were purchased from Xi'an Jiaotong University; the certificate number was SCXK2017-003. The rats were fed with a standard pellet diet and tap water ad libitum, and they were housed and maintained in $12 \mathrm{~h}$ light/ darkness with standard humidity and temperature in the laboratory. The principles of laboratory animal care guidelines, approved by the Animal Ethics Committee at the Shaanxi Academy of Traditional Chinese Medicine, were strictly followed.
2.4.2. Establishment and Grouping of the Migraine Rat Model. After an environmental adaptation period of 7 days, the rats were randomly divided into three groups, including the control group, model group, and TTNC group $(640 \mathrm{mg} / \mathrm{kg} / \mathrm{d})$. The TTNC group was given intragastric administration for 7 days, and the control group and model group were given equal volume normal saline. After the last administration of $30 \mathrm{~min}$, the rats were subcutaneously injected with nitroglycerin injection $10 \mathrm{mg} / \mathrm{kg}$ (Beijing Yimin Pharmaceutical Co., Ltd.) in the TTNC group and model group, and the control group was injected subcutaneously with equal volume of normal saline. The number of scratching and other behavioral manifestations of rats were recorded within $1 \mathrm{~h}$ after injection. When the rats showed irritability such as red ears, frequent head scratching, and cage climbing, it indicates that the modeling was successful. The rats were anesthetized with pentobarbital, blood was taken from the abdominal aorta, standing $2 \mathrm{~h}$, centrifugation at $1000 \mathrm{rpm}$ for $10 \mathrm{~min}$, and then blood serum was obtained. At the same time, the brainstem was isolated and brain tissue was obtained and collected in the EP tube. Blood serum and brain tissue were stored at $-80^{\circ} \mathrm{C}$ until used.

2.4.3. Preparation of Serum and Tissue Homogenate. Rat blood was taken from the abdominal aorta after anesthetized with pentobarbital, and serum was obtained. The brainstem was isolated, and the tissue was collected in EP tubes. Both serum and brain tissue were stored at $-80^{\circ} \mathrm{C}$ until used. A certain amount of tissue was cut and weighed. Ten times the amount of normal saline was added for further grinding (Haoyuan Technology Co., Ltd., China). After centrifuging twice at $4^{\circ} \mathrm{C}$, the supernatant was collected, and the protein quantification was determined by the BCA protein concentration assay kit (Beyotime, Shanghai, China).

2.4.4. Indexes Detection in Serum and Tissue. According to the manufacturer's protocols, the content of $\beta$-EP in blood serum was detected by commercial enzyme-linked immunosorbent assay ELISA kits. The nitrate reductase method was used to detect the level of NO in serum, strictly following NO one-step detection instructions (Nanjing Jiancheng, Nanjing, China). The HTR1A and DRD2 levels in brain tissue were detected by ELISA according to the instructions of ELISA kits. All the ELISA kits were purchased from Elabscience Biotechnology Co., Ltd.

2.4.5. Statistics Analysis. All data were expressed as the mean \pm standard deviation (SD). GraphPad Prism 5.01 software was used to ascertain statistically significant differences. The differences among multiple groups were evaluated using the one-way analysis of variance (ANOVA), and the $t$-test was used for pairwise comparison. The difference between the means was considered to be statistically significant at $P<0.05$ and $P<0.01$. 


\section{Results and Discussion}

3.1. UPLC-UESI-Q Exactive Focus Analysis of TTNC Extracts. In terms of mapping the chemical profiles of the extracts of TTNC, data of mass fragmentation coupled with highresolution spectrometry provided sufficient information. In addition, UPLC-UESI-Q Exactive Focus was used in this present study. The base peak chromatogram (BPC) of TTNC extracts in the mode of positive and negative ions is shown in Figure 2. Compounds were identified by determining the elemental compositions of the precursor and product ions. The molecular formula and rational fragmentation patterns and pathways of these compounds were then identified based on a comparison of these data with chemical databases. According to this situation, the UPLC-UESI-Q Exactive Focus method was used in combination with available databases and literature data. According to ChemSpider (https://www.chemspider.com), the mass spectrometry database (https://www.massbank.jp), and many related Chinese herbal medicine research chemical components [26-38], the chemical database was established to identify the components of TTNC. The source of chemical components in TTNC was determined by the sample injection analysis of 6 botanical drugs and the ion flow extraction from the first-level high-resolution data. Finally, a total of 88 compounds were obtained, including 29 ingredients in Angelicae Sinensis Radix, 25 ingredients in Polygoni Multiflori Radix, 13 ingredients in Gastrodiae Rhizoma, 12 ingredients in Saposhnikoviae Radix, 8 ingredients in Smilacis Glabrae Rhizoma, and 1 ingredient in Scorpio. The results are given in Table S1 in Supplemental Materials.

3.2. The Targets of the Chemical Composition for TTNC in the Treatment of Migraine. To obtain the targets of the 88 components for TTNC, target fishing was performed in accordance with the method described in Section 2.2.1. As a result, 895 targets were obtained for 50 compounds. Then, "migraine" was entered as keywords, and there were 2759 targets obtained from the GeneCards, and 963 targets were considered as the migraine targets according to relevance score. An analysis was performed using Venn online software to identify common targets between component targets and disease targets with Venn's diagrams (Figure 3(a)). Finally, 102 targets were determined as the potential targets of TTNC in treating migraine.

3.3. The PPI Network Construction and Core Targets Determination. The 102 targets of TTNC were introduced into the STRING 11.0 platform for searching the interaction between potential targets (i.e., targets of TTNC). The interaction results were introduced into the Cytoscape 3.7.2 software to draw the PPI network. Also, the nodes represented targets and the edges represented the interaction between different nodes. After removing the targets far away from the main network, there were 73 nodes and 206 edges (Figure 3(b)). The larger the circle, the brighter the color. This indicates that it plays a more important role in the network, and it will be more crucial for the occurrence of migraine and the treatment of TTNC.

3.4. The Results of KEGG Pathway Analysis. The 73 targets were mapped to a total of 124 KEGG pathways. The obviously unrelated KEGG pathways were removed, such as "pathways in cancer," "prostate cancer," "hepatitis C," and so on. Finally, the pathways with the top 20 KEGG pathways of the $\log q$-value value are shown in Figure 3(c). According to the results, the cAMP signaling pathway, PI3K-Akt signaling pathway, rap1 signaling pathway, and NADH: ubiquinone oxidoreductase, mitochondria may be the important pathways for TTNC. In these pathways, the cAMP signaling pathway was one of the most important pathways with the most enriched targets (12 targets, including ADORA1, AKT1, CFTR, CREB1, DRD2, EDNRA, HTR1A, HTR1F, NFKB1, NFKBIA, PIK3CA, and PPARA) and smaller $\log q$-value $(\log q$-value $=-1.04 E+01)$. The number of enriched targets in the PI3K-Akt signaling pathway was 12 . The $\log q$-value is $-8.52 E+00$. In addition, $\mathrm{NADH}$ : ubiquinone oxidoreductase, mitochondria have the smallest $\log q$-value $(\log q$-value $=-1.55 E+01)$, and may be one of the other most important pathways for TTNC.

\subsection{The Herbs-Compounds-Targets-Pathways (H-C-T-P)} Network Construction and Analysis. The H-C-T-P network was constructed, and analysis for TTNC in treatment with migraine was done by using Cytoscape 3.7.2 (Figure 4). The network contained 150 nodes ( 6 herbs, 51 compounds, 73 targets, and 20 pathways) and 381 edges. In Figure 4, the herbs nodes are shown in yellow diamond, the compounds nodes are shown in blue hexagon, the targets nodes are shown in purple rectangle, and the pathways nodes are shown in pink ellipse. The gray edges represented the relationships among the herb, compounds, targets, and pathways. The topological properties of the network were analyzed by the network analyzer plugin in Cytoscape 3.7.2, including degree centrality (DC), betweenness centrality (BC), and closeness centrality (CC) (Tables 1-3). These topological properties are important parameters of network analysis, which can reflect the importance of each node in the network. The higher the topological properties, the more important the compound or target is in the network.

According to the results, we obtained 18 potential key compounds, 20 potential key targets, and 6 potential key pathways with higher than the average of DC. In the key compounds, gastrodin, the main effective constitute from Gastrodiae Rhizoma, has been indicated for migraine treatment and prophylaxis more than 30 years, with demonstrated safety [39]. The research of Wang et al. showed that the antimigraine effect of gastrodin is associated with inhibition of the trigeminal pain pathway at peripheral and central sites in the model of nitroglycerin (NTG)-induced migraine in rats. The underlying mechanism of gastrodin reflected by a decrease in c-Fos expression in TNC, direct scavenging of $\mathrm{NO}$, and decrease in the subsequent peripheral release of CGRP [40]. In addition, adenosine is a purine nucleoside that plays a critical role in numerous cellular and 


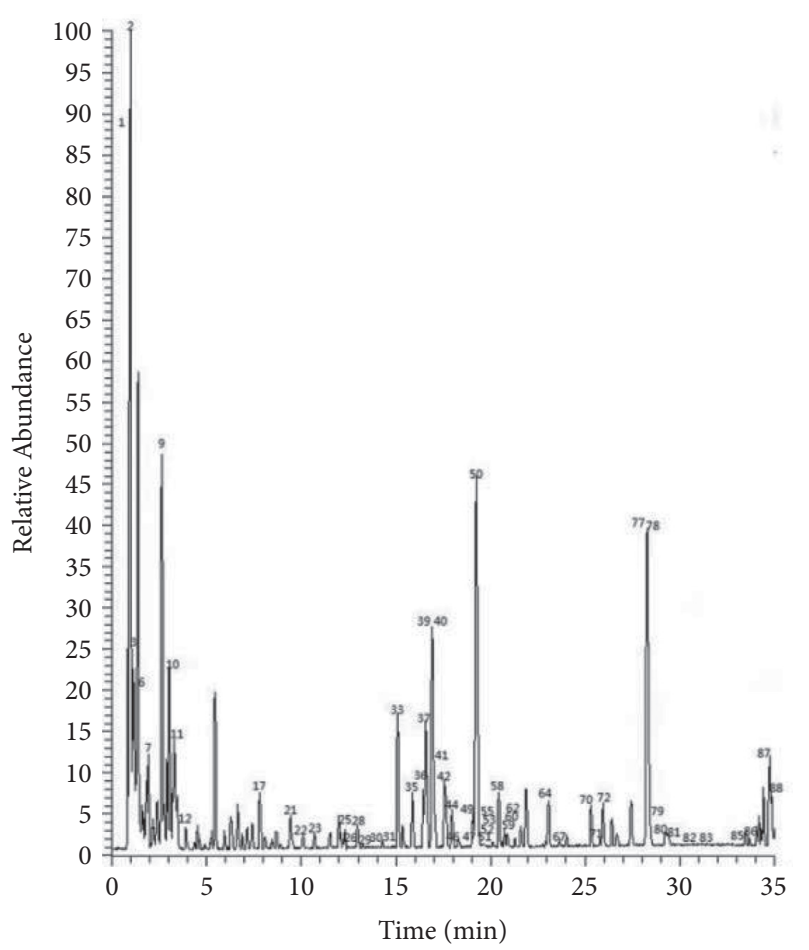

(a)

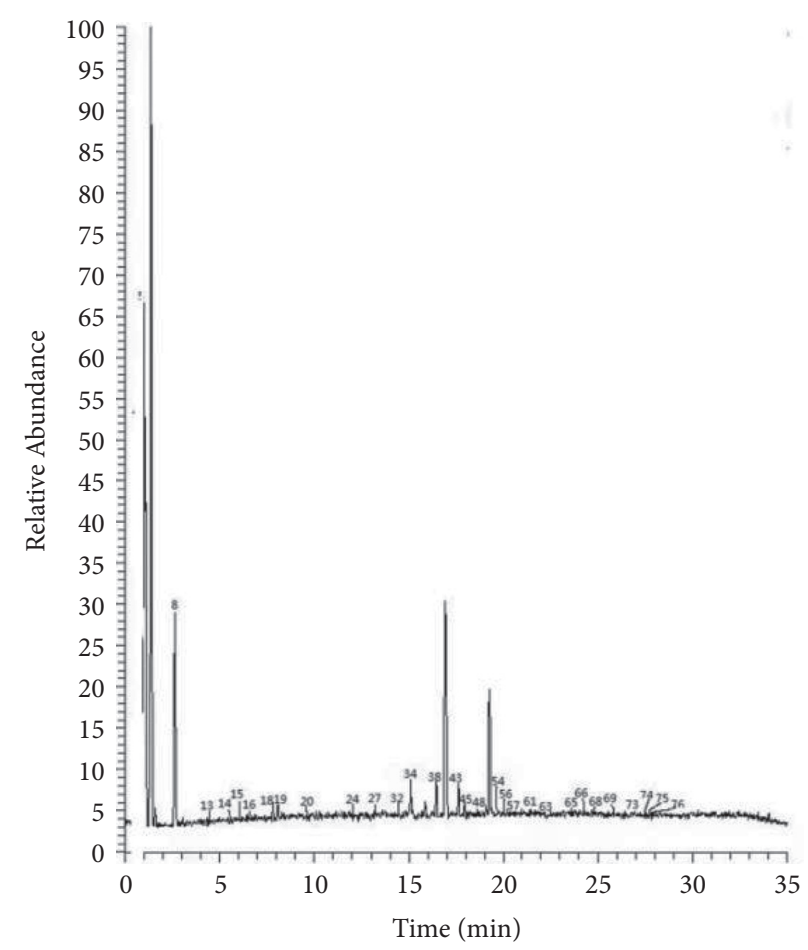

(b)

Figure 2: The total ion flow diagram of TTN. (a) The positive ion mode. (b) The negative ion mode.

molecular functions, such as the metabolism, cell signaling, purinergic neuronal signaling, and inflammation, throughout the brain. Recent studies have proved that the role of adenosine in different forms of headache, headache triggers, and basic headache physiology and suggested it was a core component to headache pain. In addition, adenosine signaling may initiate headache pain by modulation of intracellular CAMP production or AMPK activity that can change neuronal conductance within critical trigeminal pain processing brain regions [41]. Besides, some components of Saposhnikoviae Radix have also showed higher DC, BC, and CC, such as nodakenin, sec-O-glucosylhamaudol, 5-Omethylvisammioside, and prim-O-glucosylcimifugin. Saposhnikoviae Radix mainly has antipyretic, analgesic, sedative, anti-inflammatory, antibacterial, antitumor, improving immune function, antiallergy, anticoagulant, and other pharmacological effects. It is one of the commonly used medicines for headache.

In terms of the key targets, AKT1 is one of 3 closely related serine/threonine-protein kinases (AKT1, AKT2, and AKT3) called the AKT kinase, which regulates many processes including the metabolism, proliferation, cell survival, growth, and angiogenesis through serine and/or threonine phosphorylation of a range of downstream substrates [42-44]. Previous studies have revealed that the expression level of phosphorylated AKT (P-AKT) was significantly increased in a rat model group of migraine compared with the control group via measuring the p-AKT level [45]. Moreover, AKT controlled the tempo of the process of newborn neurons integration during adult neurogenesis, including correct neuron positioning, dendritic development, and synapse formation, and plays a role of key modulator in the AKT-mTOR signaling pathway [46]. MAPK8 and MAPK1 were two of member of MAPK family, and activation of MAPK is suggested to mediate the synthesis and the release of calcitonin gene-related peptide (CGRP) which has long been implicated in the pathophysiology of migraine $[47,48]$. Also, they are monoclonal antibodies (mAbs) targeting CGRP or its receptor was approved to be migraine-specific treatment in late decades [49-51]. Thus, the result in this study proved that key targets MAPK1 and MAPK8 regulating migraine through MAPK signaling are consistent with previous research. It was well known that migraine is a nervous system disease with a significant genetic predisposition [52-54]. Furthermore, sufficient studies have proved the significant role of sex hormones in migraine, and variants in the ESR1 gene were linked to migraine. It showed the association between rs2234693 in ESR1 gene and menstrually related migraine in research which contains a cohort of 533 controls and 494 migraine patients [55].

Amounts of evidences supported the significance role of the PI3K-Akt signaling pathway in migraine $[45,56]$. A study has confirmed that glucagon-like peptide-1 receptor (GLP-1R) agonist inhibited the upregulation of PI3K/p-Akt in the trigeminal nucleus caudalis (TNC) via Western blotting, which indicated that microglial GLP-1R activation in TNC may suppress the central sensitization of chronic migraine by regulating TNC microglial activation via the PI3K/Akt pathway [57]. Furthermore, PRKCA, as a core 

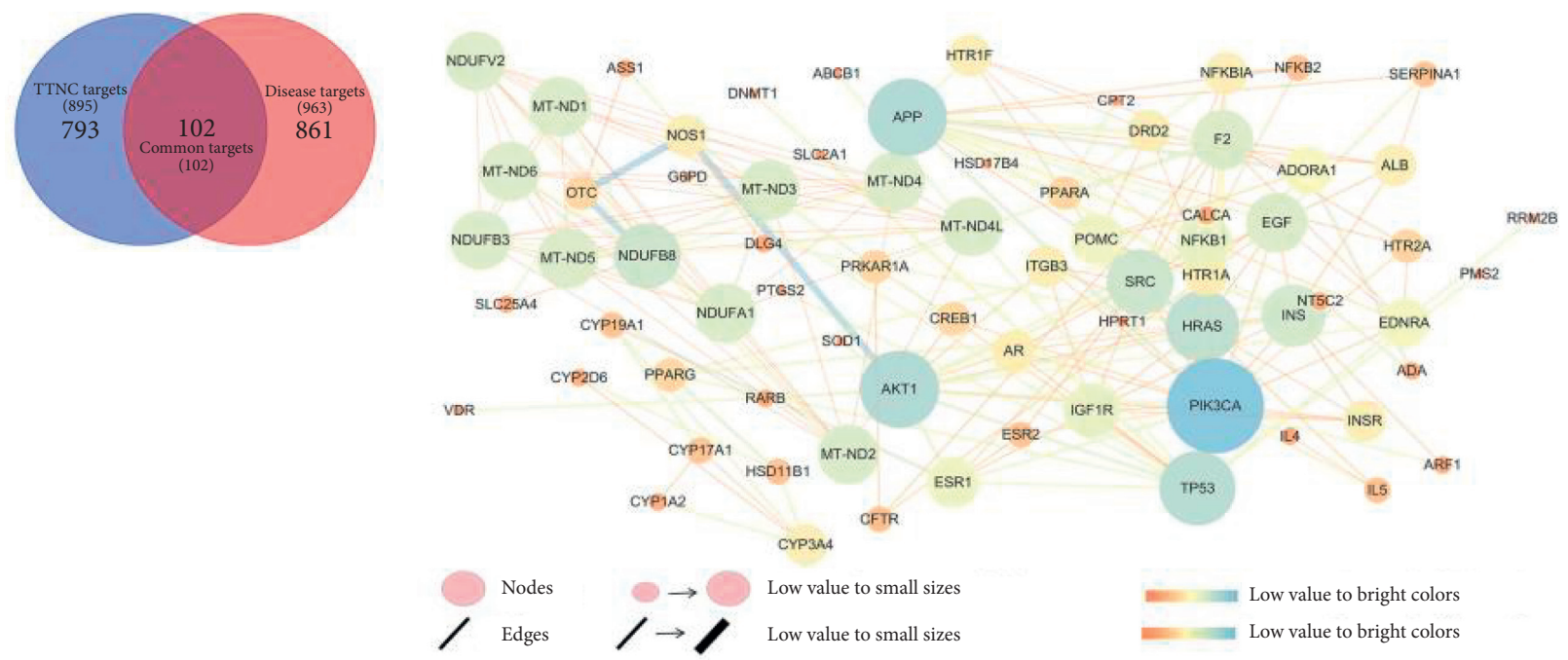

(a)

(b)

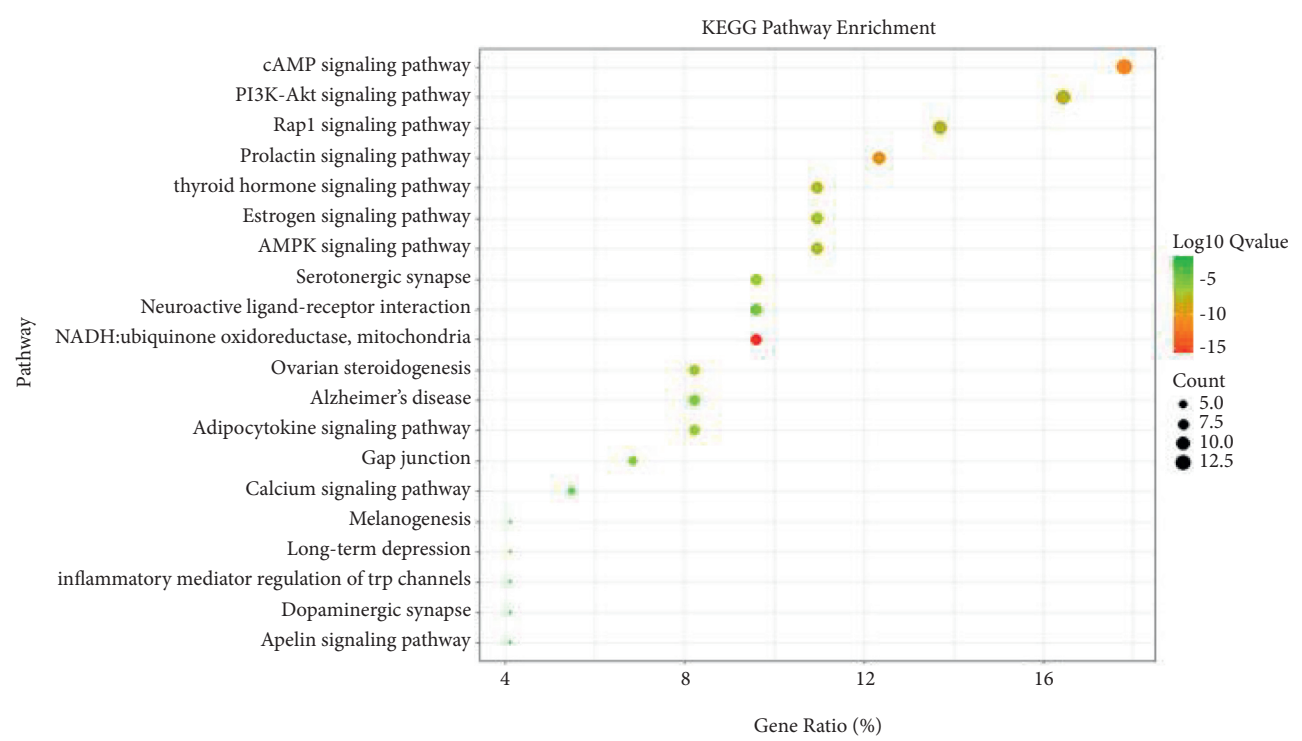

(c)

FIgURE 3: The results of Venn analysis, protein-protein interaction (PPI), and the KEGG pathway enrichment analysis. (a) The Venn's diagrams between the component targets for TTNC and disease targets for migraine. (b) The PPI network for TTNC in treatment with migraine. (c) The results of KEGG pathway enrichment analysis for TTNC in treatment with migraine.

target of our research in both the P13K-AKT pathway and MAPK signaling pathway, is calcium-activated and phospholipid and diacylglycerol (DAG)-dependent serine/threonine-protein kinase activating signaling cascade involving MAPK1/3 (ERK1/2) and RAP1GAP, thus promoting cell growth by phosphorylating and activating RAF1. It mediates the activation of the MAPK/ERK signaling cascade and/or upregulates CDKN1A, which facilitates active cyclin-dependent kinase $(\mathrm{CDK})$ complex formation in glioma cells.

3.6. Molecular Docking Results. The molecular docking was carried out to further explore the interaction between some compounds and targets. We selected some key targets that have been reported to be closely related to migraine (DRD2, HTR1A, HTR2A, ESR1, ESR2, CYP19A1, and AR) and the corresponding compounds for molecular docking. The binding energy is given in Table 4. The results of binding energy showed that the interaction between these targets and related compounds was strong. Take the binding between HTR1A and decursin as an example (Figure 5); decursin can bind in the active pocket composed of residues Val117, Phe362, Ala203, Ile189, Phe112, Asn386, Tyr390, Asp116, Cys120, and Trp358 for HTR1. There is a hydrogen bond between Thr121 and decursin, and the binding energy is $-8.65 \mathrm{kcal} / \mathrm{mol}$.

\subsection{Experimental Verification Results}

3.7.1. Behavioral Changes. As shown in Figure 6(a), compared with the control group, the incubation period of red ears and frequent head scratching in the model group was significantly 


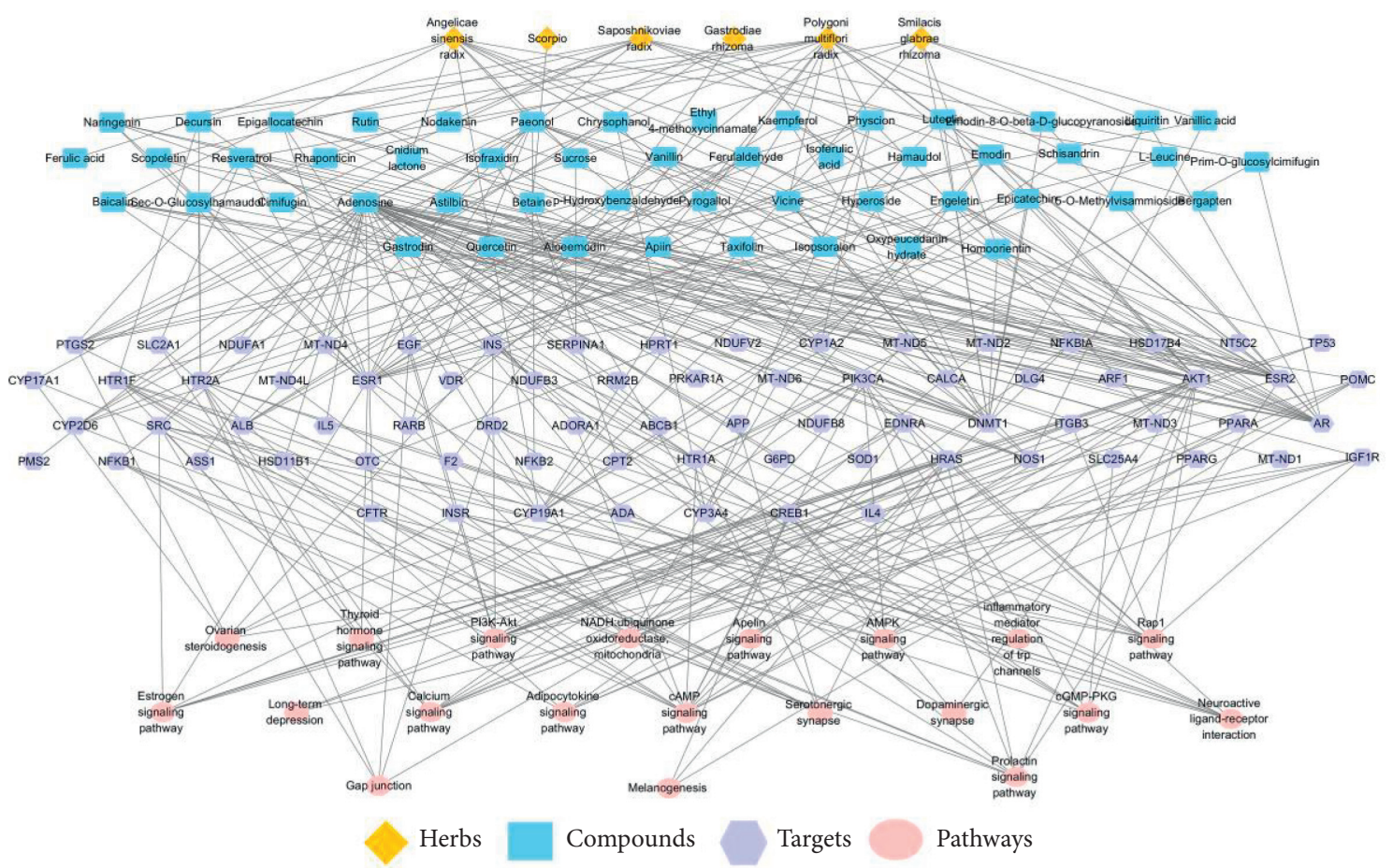

FIGURE 4: The herbs-compounds-targets-pathways (H-C-T-P) network for TTNC in treatment with migraine.

TABLE 1: The degree centrality (DC), betweenness centrality (BC), and closeness centrality (CC) values of the key compounds for TTNC in treating migraine.

\begin{tabular}{|c|c|c|c|c|c|}
\hline No. & Compound name & Source & $\mathrm{CC}$ & $\mathrm{DC}$ & $\mathrm{BC}$ \\
\hline 1 & Adenosine & Gastrodiae Rhizoma & 0.4257 & 43 & 0.4103 \\
\hline 2 & Paeonol & Angelicae Sinensis Radix & 0.3326 & 12 & 0.0750 \\
\hline 3 & Resveratrol & Smilacis Glabrae Rhizoma & 0.3435 & 9 & 0.0334 \\
\hline 4 & Emodin & Polygoni Multiflori Radix & 0.3501 & 7 & 0.0374 \\
\hline 5 & Physcion & Polygoni Multiflori Radix & 0.2792 & 7 & 0.0231 \\
\hline 6 & Astilbin & Smilacis Glabrae Rhizoma & 0.3468 & 6 & 0.0111 \\
\hline 7 & Baicalin & Angelicae Sinensis Radix & 0.3623 & 6 & 0.0333 \\
\hline 8 & Engeletin & Smilacis Glabrae Rhizoma & 0.3468 & 6 & 0.0111 \\
\hline 9 & Epicatechin & Polygoni Multiflori Radix & 0.3468 & 6 & 0.0101 \\
\hline 10 & Epigallocatechin & Polygoni Multiflori Radix & 0.3468 & 6 & 0.0101 \\
\hline 11 & Ferulaldehyde & Angelicae Sinensis Radix & 0.2961 & 6 & 0.0161 \\
\hline 12 & Homoorientin & Smilacis Glabrae Rhizoma & 0.3341 & 6 & 0.0078 \\
\hline 13 & Kaempferol & Polygoni Multiflori Radix & 0.3468 & 6 & 0.0101 \\
\hline 14 & Luteolin & Polygoni Multiflori Radix & 0.3468 & 6 & 0.0101 \\
\hline 15 & Naringenin & Polygoni Multiflori Radix & 0.3468 & 6 & 0.0101 \\
\hline 16 & Quercetin & Angelicae Sinensis Radix & 0.3518 & 6 & 0.0282 \\
\hline 17 & Taxifolin & Smilacis Glabrae Rhizoma & 0.3372 & 6 & 0.0061 \\
\hline 18 & Vanillin & Angelicae Sinensis Radix & 0.3587 & 6 & 0.0290 \\
\hline
\end{tabular}

shortened. In contrast, the number of head scratching was significantly increased within $1 \mathrm{~h}$ after nitroglycerin injection $(P<0.05)$. Compared with the model group, the incubation period was significantly prolonged, and the number of head scratching was significantly decreased in the TTNC group $(P<0.05)$. The results showed that TTNC can improve the behavioral changes of migraine rats.

3.7.2. The Content Detection of NO and $\beta$-EP in Blood Serum. Figure 6(b) shows the content detection results of $\mathrm{NO}$ and $\beta$-EP in blood serum. The content of NO in the animal serum model group increased significantly compared with the control group $(P<0.05)$, indicating that the model was successful. Compared with the model group, the content of NO in the TTNC group was significantly decreased $(P<0.05)$. It is well known that NO plays a key role in the mechanism of migraine and other vascular headache. NO can expand blood vessels, result in neurogenic inflammation, activate the sensitivity of injured sensory neurons, and mediate pain signal transmission of the body and lead to migraine. The experiment in the present study is a classic migraine model. After injection 
TABLE 2: The degree centrality (DC), betweenness centrality (BC), and closeness centrality (CC) values of the key targets for TTNC in treating migraine.

\begin{tabular}{|c|c|c|c|c|c|}
\hline No. & Gene name & Target name & $\mathrm{CC}$ & $\mathrm{DC}$ & $\mathrm{BC}$ \\
\hline 1 & AKT1 & AKT serine/threonine kinase 1 & 0.4345 & 24 & 0.1875 \\
\hline 2 & ESR1 & Estrogen receptor 1 & 0.3544 & 20 & 0.0592 \\
\hline 3 & ESR2 & Estrogen receptor 2 & 0.3427 & 18 & 0.0394 \\
\hline 4 & $\mathrm{AR}$ & Androgen receptor & 0.3318 & 18 & 0.0887 \\
\hline 5 & DNMT1 & DNA methyltransferase 1 & 0.3862 & 18 & 0.1141 \\
\hline 6 & HRAS & HRas proto-oncogene, GTPase & 0.3596 & 11 & 0.0450 \\
\hline 7 & CYP19A1 & Cytochrome P450 family 19 subfamily a member 1 & 0.2967 & 10 & 0.0123 \\
\hline 8 & NOS1 & Nitric oxide synthase, brain & 0.3782 & 10 & 0.0583 \\
\hline 9 & HTR1F & 5-Hydroxytryptamine receptor $1 \mathrm{~F}$ & 0.2908 & 9 & 0.0112 \\
\hline 10 & HTR2A & 5-Hydroxytryptamine receptor $2 \mathrm{~A}$ & 0.2920 & 9 & 0.0192 \\
\hline 11 & PIK3CA & Phosphatidylinositol 4,5-bisphosphate 3-kinase catalytic subunit alpha isoform & 0.3510 & 9 & 0.0241 \\
\hline 12 & PTGS2 & Prostaglandin G/H synthase 2 & 0.3349 & 9 & 0.0471 \\
\hline 13 & CREB1 & Cyclic AMP-responsive element-binding protein 1 & 0.3460 & 8 & 0.0187 \\
\hline 14 & HTR1A & 5-Hydroxytryptamine receptor $1 \mathrm{~A}$ & 0.3042 & 8 & 0.0220 \\
\hline 15 & INS & Insulin & 0.2920 & 7 & 0.0089 \\
\hline 16 & SRC & Proto-oncogene tyrosine-protein kinase Src & 0.3333 & 7 & 0.0160 \\
\hline 17 & DRD2 & $\mathrm{D}(2)$ dopamine receptor & 0.3364 & 6 & 0.0205 \\
\hline 18 & EDNRA & Endothelin-1 receptor & 0.3160 & 6 & 0.0127 \\
\hline 19 & IGF1R & Insulin-like growth factor 1 receptor & 0.3318 & 6 & 0.0117 \\
\hline 20 & INSR & Insulin receptor & 0.3333 & 6 & 0.0105 \\
\hline
\end{tabular}

TABLE 3: The degree centrality (DC), betweenness centrality (BC), and closeness centrality (CC) values of the key pathways for TTNC in treating migraine.

\begin{tabular}{|c|c|c|c|c|c|}
\hline No. & Pathway & Targets & CC & DC & $\mathrm{BC}$ \\
\hline 1 & cAMP signaling pathway & $\begin{array}{c}\text { ADORA1, AKT1, CFTR, CREB1, DRD2, EDNRA, HTR1A, HTR1F, NFKB1, } \\
\text { NFKBIA, PIK3CA, PPARA }\end{array}$ & 0.3436 & 12 & 0.0524 \\
\hline 2 & $\begin{array}{r}\text { Retrograde e } \\
\text { sig }\end{array}$ & $\begin{array}{l}\text { ND1, ND2, ND3, ND4, ND4L, ND5, ND6, NDUFA1, NDUFB3, NDUFB8, } \\
\text { NDUFV2, PTGS2 }\end{array}$ & 0.3085 & 12 & 0.0321 \\
\hline 3 & PI3K-Akt signa & $\begin{array}{c}\text { AKT1, CREB1, EGF, HRAS, IGF1R, IL4, INS, INSR, ITGB3, NFKB1, } \\
\text { PIK3CA, TP53 }\end{array}$ & 0.3356 & 12 & 0.0394 \\
\hline 4 & & AKT1, DRD2, EGF, HRAS, IGF1R, INS, INSR, ITGB3, PIK3CA, SRC & & 10 & 0.0 \\
\hline 5 & $\operatorname{trc}$ & $\mathrm{C}$ & & 8 & 0.026 \\
\hline 6 & cGMP-PKG signaling pathway & ADORA1, AKT1, SLC25A4, CREB1, EDNRA, INS, INSR & 0.3208 & 7 & 0.0125 \\
\hline
\end{tabular}

TABLE 4: The docking energy results of the complex between key targets and the key compounds for TTNC in treatment with migraine.

\begin{tabular}{lccccc}
\hline Protein name & Gene name & PDB ID & Ligand name & PubChem ID & Binding energy (kcal/mol) \\
\hline 5-Hydroxytryptamine receptor 1A & HTR1A & 7E2Z & Decursin & 442126 & -8.65 \\
5-Hydroxytryptamine receptor 2A & HTR2A & 6WGT & Hamaudol & 164722 & -7.94 \\
D(2) dopamine receptor & DRD2 & 6CM4 & Adenosine & 60961 & -6.32 \\
Nitric oxide synthase, brain & NOS1 & 3HSN & Adenosine & 60961 & -5.06 \\
Estrogen receptor & ESR1 & 5FQP & Epicatechin & 72276 & -7.70 \\
Estrogen receptor beta & ESR2 & 2YLY & Epigallocatechin & 72277 & -6.74 \\
Aromatase & CYP19A1 & 3S79 & Kaempferol & 5280863 & -6.88 \\
Androgen receptor & AR & 2PIW & Paeonol & 11092 & -4.51 \\
\hline
\end{tabular}

of nitroglycerin, the content of NO in animal blood increase rapidly, resulting in migraine. In the TTNC group, the content of NO decreased significantly, indicating that TTNC can downregulate the production of NO induced by nitroglycerin, so as to alleviate the attack of migraine. In addition, the content of $\beta$-EP in the model group decreased significantly compared with the control group $(P<0.05)$, while the content of $\beta$-EP in the TTNC group increased significantly compared with the model group
$(P<0.05) . \beta$-EP is $\alpha$-endogenous opioid peptide with a strong analgesic effect. In the case of disorders of the endorphin system in the body, $\beta$-EP release decreased immediately, the pain threshold of body also decreased, and migraine are more likely to occur. In this experiment, NO induced endogenous opioid peptide system disorder, and the content of $\beta$-EP decreased. After the intervention of TTNC, the content of $\beta$-EP increased, indicating that TTNC could upregulate the level of $\beta$-EP induced by 


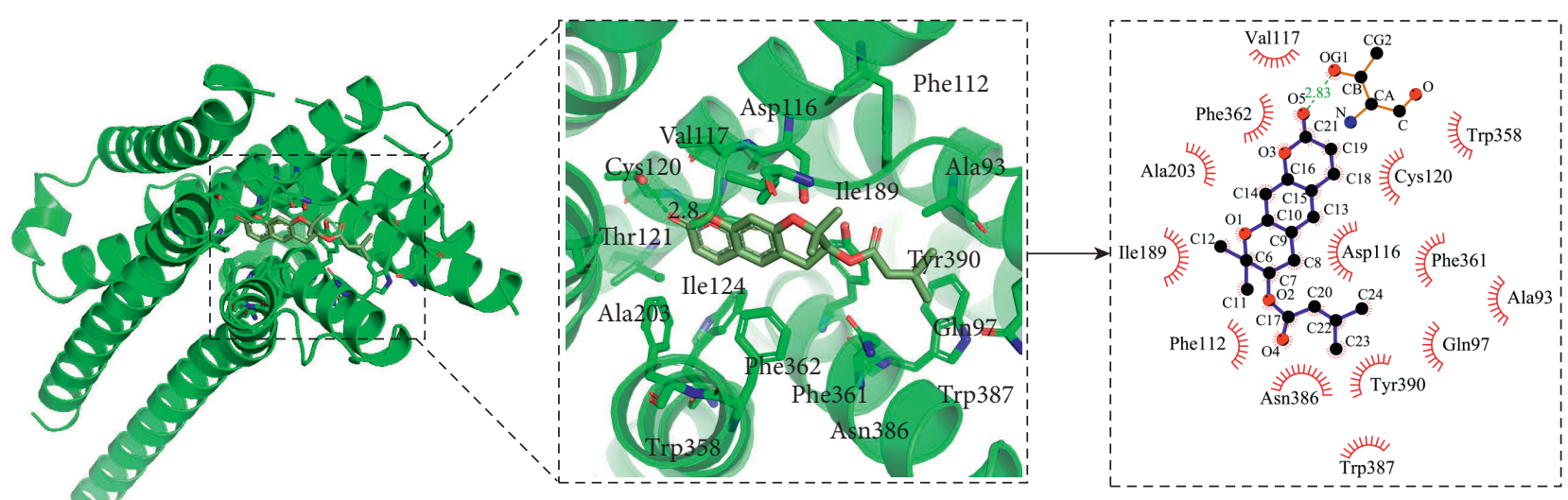

(a)

(b)

(c)

FIgURE 5: The molecular docking results of TTNC in treatment with migraine.
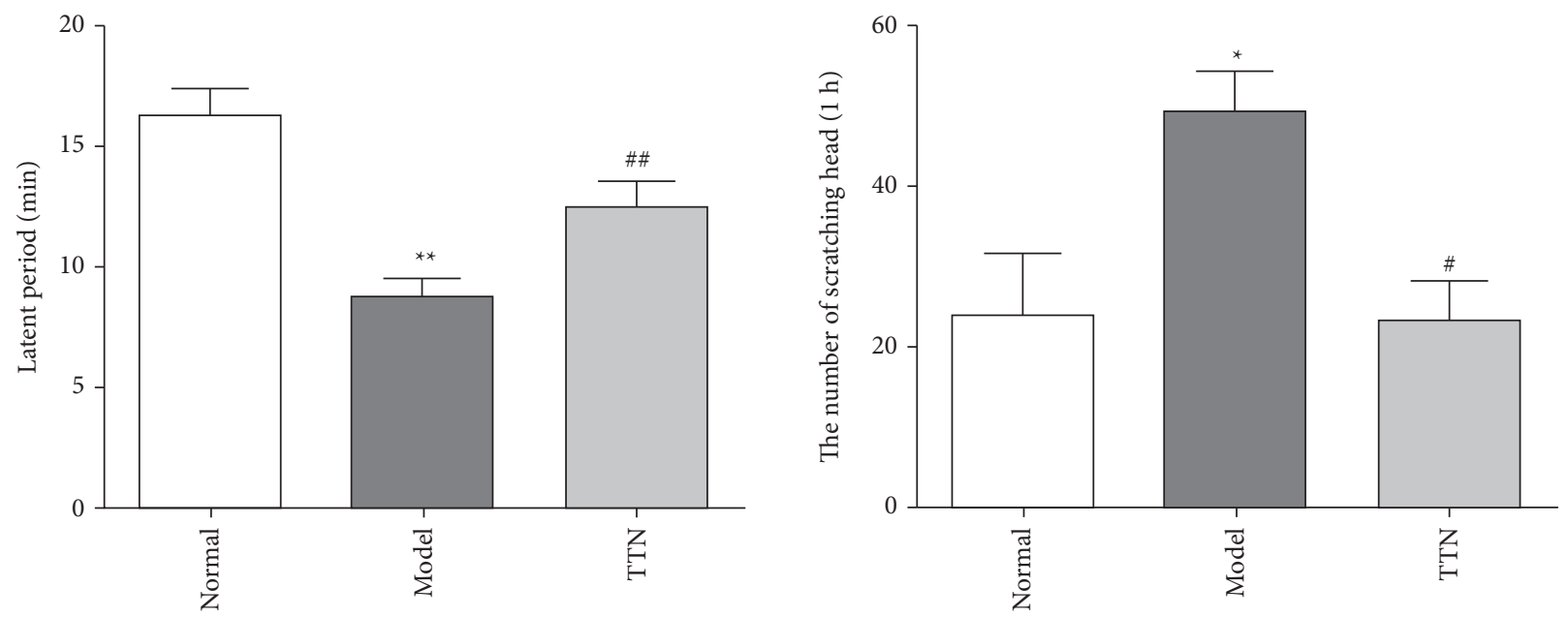

(a)
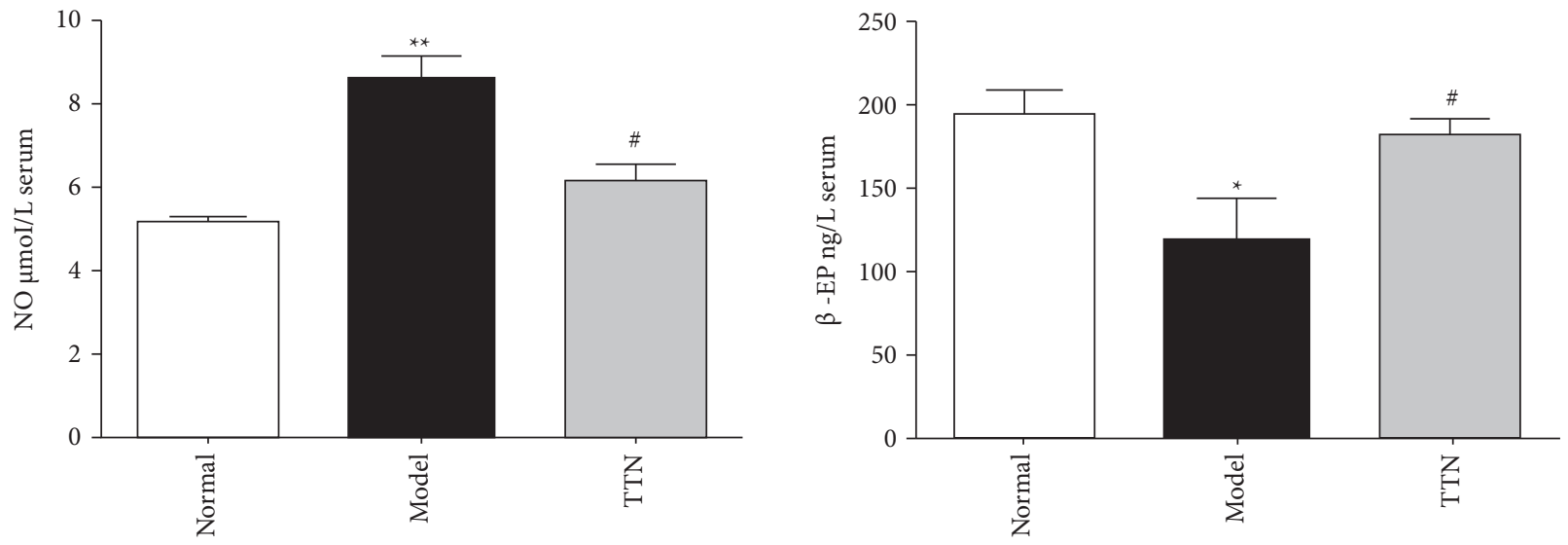

(b)

Figure 6: Continued. 



(c)

Figure 6: The results of experimental verification. (a) Behavioral changes of the control, model, and TTN group. (b) The contents of NO and $\beta$-EP in blood serum of the control, model, and TTN group. (c) The HTR1A and DRD2 levels in brain tissue detected by ELISA of the control, model, and TTN group.

nitroglycerin, so as to relieve migraine. These results showed that TTNC has a good effect on migraine induced by nitroglycerin.

\subsubsection{The HTR1A and DRD2 Levels in Brain Tissue Detected} by ELISA. The two key targets of HTR1A and DRD2 in brain tissue were verified in vivo by ELISA detection (Figure 6(c)) for the sake of verifying the results of previous prediction after comprehensively considering the results of molecular docking. The results showed that the level of HTR1A of the model group was significantly lower than that of the control group $(P<0.05)$, and the level was significantly higher in the TTNC group compared with the model group $(P<0.05)$. Besides, the level of DRD2 in brain tissue of the model group was significantly lower than that of the control group $(P<0.01)$, and compared with the model group, the level of DRD2 treated with TTNC improved significantly $(P<0.01)$.

\section{Conclusion}

In the present study, a systematical research strategy was adopted to investigate the mechanism of TTNC in treatment with migraine. It contained UPLC-UESI-Q Exactive Focus, network pharmacology, and in vivo experiment. Also, the target fishing, protein-protein interaction (PPI), KEGG pathway enrichment analysis, and herbs-compounds-targets-pathways (H-C-T-P) network construction were performed according to the network pharmacology to predict the potential pharmacodynamic components, targets, and pathways. After 18 potential key compounds, 20 potential key targets and 6 potential signaling pathways were obtained for TTNC in treatment with migraine. Finally, molecular docking and experimental were carried out to verify some key targets. More experimental verification and research still need to be carried out for TTNC in treatment with migraine. The results of this study may provide a theoretical basis for further research on the molecular mechanism of TTNC in the treatment of migraine.

\section{Data Availability}

The data used to support the findings of this study are included within the article and Supplementary Materials.

\section{Conflicts of Interest}

The authors declare that they have no conflicts of interest.

\section{Authors' Contributions}

Xia Du and Zhibiao Di contributed equally to this work.

\section{Acknowledgments}

This work was supported by the study of Key Research and Development Program of Shaanxi (2018ZDXM-SF-003).

\section{Supplementary Materials}

Table S1: the information of 88 ingredients of TTNC by UPLC-UESI-Q Exactive Focus analysis. (Supplementary Materials)

\section{References}

[1] International Headache Society, "The international classification of headache disorders," Cephalalgia, vol. 38, pp. 1-211, 2018.

[2] D. W. Dodick, "Migraine," The Lancet, vol. 391, no. 10127, pp. 1315-1330, 2018.

[3] GBD 2015 Disease and Injury Incidence and Prevalence Collaborators, "Global, regional, and national incidence, prevalence, and years lived with disability for 310 diseases and injuries, 1990-2015: a systematic analysis for the Global Burden of Disease Study 2015," Lancet, vol. 388, no. 10053, pp. 1545-1602, 2016. 
[4] GBD 2015 Neurological Disorders Collaborator Group, "Global, regional, and national burden of neurological disorders during 1990-2015: a systematic analysis for the Global Burden of Disease Study 2015," The Lancet Neurology, vol. 16, no. 11, pp. 877-897, 2017.

[5] GBD 2017 Disease and Injury Incidence and Prevalence Collaborators, "Global, regional, and national incidence, prevalence, and years lived with disability for 354 diseases and injuries for 195 countries and territories, 1990-2017: a systematic analysis for the Global Burden of Disease Study 2017," Lancet, vol. 392, no. 10159, pp. 1789-1858, 2018.

[6] S. K. Aurora and M. F. Brin, "Chronic migraine: an update on physiology, imaging, and the mechanism of action of two available pharmacologic therapies," The Journal of Head and Face Pain, vol. 57, no. 1, pp. 109-125, 2017.

[7] A. Moutal, Y. Wang, X. Yang et al., "Dissecting the role of the CRMP2-neurofibromin complex on pain behaviors," Pain, vol. 158, no. 11, pp. 2203-2221, 2017.

[8] M. D. Ferrari, M. Färkkilä, U. Reuter et al., "Acute treatment of migraine with the selective 5-HT1F receptor agonist lasmiditan-a randomised proof-of-concept trial," Cephalalgia, vol. 30, no. 10, pp. 1170-1178, 2010.

[9] C. Chan and P. J. Goadsby, "Recent advances in pharmacotherapy for episodic migraine," CNS Drugs, vol. 33, no. 11, pp. 1053-1071, 2019.

[10] C. Ayata, H. Jin, C. Kudo, T. Dalkara, and M. A. Moskowitz, "Suppression of cortical spreading depression in migraine prophylaxis," Annals of Neurology, vol. 59, no. 4, pp. 652-661, 2006.

[11] F. Li, E. Qiu, Z. Dong, R. Liu, S. Wu, and S. Yu, "Protection of flunarizine on cerebral mitochondria injury induced by cortical spreading depression under hypoxic conditions," The Journal of Headache and Pain, vol. 12, no. 1, pp. 47-53, 2011.

[12] A. Tozzi, A. de Iure, M. Di Filippo et al., "Critical role of calcitonin gene-related peptide receptors in cortical spreading depression," Proceedings of the National Academy of Sciences, vol. 109, no. 46, pp. 18985-18990, 2012.

[13] S. Yu, Y. Ran, Q. Wan et al., "Efficacy and safety of Toutongning capsule in patients with migraine: a multicenter, randomized, double-Blind, placebo-controlled trial," Journal of Alternative \& Complementary Medicine, vol. 25, no. 12, pp. 1215-1224, 2019.

[14] S. Li and B. Zhang, "Traditional Chinese medicine network pharmacology: theory, methodology and application," Chinese Journal of Natural Medicines, vol. 11, no. 2, pp. 110-120, 2013.

[15] H. Li, L. Zhao, B. Zhang et al., "A network pharmacology approach to determine active compounds and action mechanisms of ge-gen-qin-lian decoction for treatment of type 2 diabetes," Evidence-based Complementary and Alternative Medicine, vol. 2014, Article ID 495840, 12 pages, 2014.

[16] J. Wang, Z. Jia, Z. Zhang et al., "Analysis of chemical constituents of melastoma dodecandrum Lour. by UPLC-ESI-Qexactive focus-MS/MS," Molecules, vol. 22, no. 3, Article ID 476, 2017.

[17] Y. Wang, Y. Han, Y. Xie, P. Xu, and W. Li, "The metabolism distribution and effect of dinotefuran in Chinese lizards (Eremias argus)," Chemosphere, vol. 211, pp. 591-599, 2018.

[18] H.-Y. Xu, Y.-Q. Zhang, Z.-M. Liu et al., "ETCM: an encyclopaedia of traditional Chinese medicine," Nucleic Acids Research, vol. 47, no. D1, pp. D976-D982, 2019.

[19] D. S. Wishart, Y. D. Feunang, A. C. Guo et al., "DrugBank 5.0: a major update to the DrugBank database for 2018," Nucleic Acids Research, vol. 46, no. D1, pp. D1074-D1082, 2018.
[20] G. Stelzer, N. Rosen, I. Plaschkes et al., "The GeneCards suite: from gene data mining to disease genome sequence analyses," Current protocols in bioinformatics, vol. 54, pp. 1-33, 2016.

[21] UniProt Consortium, "UniProt: a worldwide hub of protein knowledge," Nucleic Acids Research, vol. 47, no. D1, pp. D506-D515, 2019.

[22] D. Szklarczyk, A. L. Gable, D. Lyon et al., "STRING v11: protein-protein association networks with increased coverage, supporting functional discovery in genome-wide experimental datasets," Nucleic Acids Research, vol. 47, no. D1, pp. D607-D613, 2019.

[23] P. Shannon, A. Markiel, O. Ozier et al., "Cytoscape: a software environment for integrated models of biomolecular interaction networks," Genome Research, vol. 13, no. 11, pp. 2498-2504, 2003.

[24] D. W. Huang, B. T. Sherman, and R. A. Lempicki, "Systematic and integrative analysis of large gene lists using DAVID bioinformatics resources," Nature Protocols, vol. 4, no. 1, pp. 44-57, 2009.

[25] D. W. Huang, B. T. Sherman, and R. A. Lempicki, "Bioinformatics enrichment tools: paths toward the comprehensive functional analysis of large gene lists," Nucleic Acids Research, vol. 37, no. 1, pp. 1-13, 2009.

[26] X. Ye, Y. Wang, J. Zhao et al., "Identification and characterization of key chemical constituents in processed Gastrodia elata using UHPLC-MS/MS and chemometric methods," Journal of Analytical Methods in Chemistry, vol. 2019, Article ID 4396201, 10 pages, 2019.

[27] Y. Li, X. Q. Liu, S. S. Liu, D. H. Liu, X. Wang, and Z. M. Wang, "Transformation mechanisms of chemical ingredients in steaming process of gastrodia elata blume," Molecules, vol. 24, no. 17, Article ID 3159, 2019.

[28] J. L. Ríos, "Chemical constituents and pharmacological properties of Poria cocos," Planta Medica, vol. 77, no. 7, pp. 681-691, 2011.

[29] L.-X. Zhu, J. Xu, Y. Wu et al., "Comparative quality of the forms of decoction pieces evaluated by multidimensional chemical analysis and chemometrics: poria cocos, a pilot study," Journal of Food and Drug Analysis, vol. 27, no. 3, pp. 766-777, 2019.

[30] J. X. Zhang and Y. M. Cui, "Chemical constituents from Polygonum multiflorum," China Journal of Chinese Materia Medica, vol. 41, no. 17, pp. 3252-3255, 2016.

[31] J.-B. Yang, Y. Liu, Q. Wang et al., "Characterization and identification of the chemical constituents of Polygonum multiflorum Thunb. by high-performance liquid chromatography coupled with ultraviolet detection and linear ion trap FT-ICR hybrid mass spectrometry," Journal of Pharmaceutical and Biomedical Analysis, vol. 172, pp. 149-166, 2019.

[32] W.-L. Wei, R. Zeng, C.-M. Gu, Y. Qu, and L.-F. Huang, "Angelica sinensis in China-A review of botanical profile, ethnopharmacology, phytochemistry and chemical analysis," Journal of Ethnopharmacology, vol. 190, pp. 116-141, 2016.

[33] K. Zhang, M. Yan, S. Han et al., "Identification of chemical markers for the discrimination of Radix Angelica sinensis grown in Geoherb and Non-Geoherb Regions Using UHPLCQTOF-MS/MS based metabolomics," Molecules, vol. 24, no. 19, Article ID 3536, 2019.

[34] S. Zhang, B. He, J. Ge et al., "Extraction, chemical analysis of Angelica sinensis polysaccharides and antioxidant activity of the polysaccharides in ischemia-reperfusion rats," International Journal of Biological Macromolecules, vol. 47, no. 4, pp. 546-550, 2010. 
[35] M. Nishihara, K. Nukui, Y. Osumi, and H. Shiota, "Quality evaluation of Saposhnikoviae radix (differences between wildtype and cultivated products)," Yakugaku Zasshi, vol. 138, no. 4, pp. 571-579, 2018.

[36] L. Chen, X. Chen, L. Su, Y. Jiang, and B. Liu, "Rapid characterisation and identification of compounds in Saposhnikoviae Radix by high-performance liquid chromatography coupled with electrospray ionisation quadrupole time-of-flight mass spectrometry," Natural Product Research, vol. 32, no. 8, pp. 898-901, 2018.

[37] J. I. Cid-Uribe, C. E. Santibáñez-López, E. P. Meneses et al., "The diversity of venom components of the scorpion species Paravaejovis schwenkmeyeri (Scorpiones: vaejovidae) revealed by transcriptome and proteome analyses," Toxicon, vol. 151, pp. 47-62, 2018.

[38] Y. Yoshimoto, M. Miyashita, M. Abdel-Wahab, M. Sarhan, Y. Nakagawa, and H. Miyagawa, "Isolation and characterization of insecticidal toxins from the venom of the north African scorpion, buthacus leptochelys," Toxins, vol. 11, no. 4, Article ID 236, 2019.

[39] Y. Q. Xiao, L. Li, and X. L. You, "Studies on chemical constituents of effective part of gastrodia elata," China Journal of Chinese Materia Medica, vol. 27, no. 1, pp. 35-36, 2002.

[40] P.-H. Wang, L.-X. Zhao, J.-Y. Wan et al., "Pharmacological characterization of a novel gastrodin derivative as a potential anti-migraine agent," Fitoterapia, vol. 109, pp. 52-57, 2016.

[41] N. T. Fried, M. B. Elliott, and M. L. Oshinsky, "The Role of adenosine signaling in headache: a Review," Brain Sciences, vol. 7, no. 3, Article ID 30, 2017.

[42] L. Rönnstrand, "Signal transduction via the stem cell factor receptor/c-Kit," Cellular and Molecular Life Sciences: CMLS, vol. 61, no. 19-20, pp. 2535-2548, 2004.

[43] K. M. Nicholson and N. G. Anderson, "The protein kinase B/ Akt signalling pathway in human malignancy," Cellular Signalling, vol. 14, no. 5, pp. 381-395, 2002.

[44] I. Hers, E. E. Vincent, and J. M. Tavaré, "Akt signalling in health and disease," Cellular Signalling, vol. 23, no. 10, pp. 1515-1527, 2011.

[45] Y.-Y. Liu, Z.-Y. Jiao, W. Li, and Q. Tian, "PI3K/AKT signaling pathway activation in a rat model of migraine," Molecular Medicine Reports, vol. 16, no. 4, pp. 4849-4854, 2017.

[46] J. M. Bristow, M. H. Sellers, D. Majumdar, B. Anderson, L. Hu, and D. J. Webb, "The Rho-family GEF Asef2 activates Rac to modulate adhesion and actin dynamics and thereby regulate cell migration," Journal of Cell Science, vol. 122, no. 24, pp. 4535-4546, 2009.

[47] O. E. Blacque and M. R. Leroux, "Bardet-Biedl syndrome: an emerging pathomechanism of intracellular transport," Cellular and Molecular Life Sciences, vol. 63, no. 18, pp. 21452161, 2006.

[48] M. G. Buzzi and M. A. Moskowitz, "The pathophysiology of migraine: year 2005," The Journal of Headache and Pain, vol. 6, no. 3, pp. 105-111, 2005.

[49] P. J. Goadsby, U. Reuter, Y. Hallström et al., "A controlled trial of erenumab for episodic migraine," New England Journal of Medicine, vol. 377, no. 22, pp. 2123-2132, 2017.

[50] S. D. Silberstein, D. W. Dodick, M. E. Bigal et al., "Fremanezumab for the preventive treatment of chronic migraine," New England Journal of Medicine, vol. 377, no. 22, pp. 2113-2122, 2017.

[51] R. Cady and R. B. Lipton, "Qualitative change in migraine prevention?" Headache: The Journal of Head and Face Pain, vol. 58, no. 7, pp. 1092-1095, 2018.
[52] A. J. Rodriguez-Acevedo, B. H. Maher, R. A. Lea, M. Benton, and L. R. Griffiths, "Association of oestrogen-receptor gene (ESR1) polymorphisms with migraine in the large Norfolk Island pedigree," Cephalalgia, vol. 33, no. 14, pp. 1139-1147, 2013.

[53] G. Joshi, S. Pradhan, and B. Mittal, "Role of the oestrogen receptor (ESR1 PvuII and ESR1 $325 \mathrm{C} \longrightarrow \mathrm{G}$ ) and progesterone receptor (PROGINS) polymorphisms in genetic susceptibility to migraine in a North Indian population," Cephalalgia, vol. 30, no. 3, pp. 311-320, 2010.

[54] A. Oterino, M. Toriello, A. Cayón et al., "Multilocus analyses reveal involvement of theESR1,ESR2, andFSHRGenes in migraine," Headache: The Journal of Head and Face Pain, vol. 48, no. 10, pp. 1438-1450, 2008.

[55] X. An, J. Fang, Q. Lin, C. Lu, Q. Ma, and H. Qu, "New evidence for involvement of ESR1 gene in susceptibility to Chinese migraine," Journal of Neurology, vol. 264, no. 1, pp. 81-87, 2017.

[56] E. O. Adewuyi, Y. Sapkota, International Endogene Consortium Iec and Me Research Team et al., "Shared molecular genetic mechanisms underlie endometriosis and migraine comorbidity," Genes, vol. 11, no. 3, Article ID 268, 2020.

[57] F. Jing, Q. Zou, Y. Wang, Z. Cai, and Y. Tang, "Activation of microglial GLP-1R in the trigeminal nucleus caudalis suppresses central sensitization of chronic migraine after recurrent nitroglycerin stimulation," The Journal of Headache and Pain, vol. 22, no. 1, Article ID 86, 2021. 